Noname manuscript No.

(will be inserted by the editor)

\title{
Grounding and Auto-abstraction
}

\section{Luca Zanetti}

Received: date / Accepted: date

\begin{abstract}
Abstraction principles and grounding can be combined in a natural way ([Ros10, 117]; [Sch11, 362]). However, some ground-theoretic abstraction principles entail that there are circles of partial ground ([Don17, 793]). I call this problem auto-abstraction. In this paper I sketch a solution. Sections 1 and 2 are introductory. In section 3 I start comparing different solutions to the problem. In section $4 \mathrm{I}$ contend that the thesis that the right-hand side of an abstraction principle is (metaphysically) prior to its left-hand side motivates an independence constraint, and that this constraint leads to predicative restrictions on the acceptable instances of ground-theoretic abstraction principles. In section $5 \mathrm{I}$ argue that auto-abstraction is acceptable unless the left-hand side is essentially grounded by the right-hand side. In section 6 I highlight several parallelisms between auto-abstraction and the puzzles of ground. I finally compare my solution with the strategies listed in section 3 .
\end{abstract}

Keywords Abstraction Principles · Grounding · Auto-abstraction · Aristotelianism · Puzzles of Ground

An earlier version of this paper was presented at the FilMat Symposium on Abstractionism in the Philosophy of Mathematics at the XII Conference of the Italian Society for Analytic Philosophy (SIFA), 4-7 September 2018, Novara. I wish to thank the organizers, Francesca Boccuni and Andrea Sereni, Øystein Linnebo, and all attendees for valuable feedback. I would also like to thank Jack Woods and three anonymous referees at Synthese for helpful suggestions.

L. Zanetti

NEtS Center, Department of Humanities and Life Sciences

Scuola Universitaria Superiore IUSS Pavia; Palazzo del Broletto, Piazza della Vittoria, 15, 27100 Pavia; Italy

E-mail: luca.zanetti@iusspavia.it 


\section{Introduction}

The two leading actors in this paper will be abstraction principles and grounding.

An abstraction principle is a universally quantified biconditional of the following form:

$$
\forall \alpha \forall \beta(\operatorname{Abs}(\alpha)=\operatorname{Abs}(\beta) \leftrightarrow \alpha \sim \beta)
$$

where $\alpha$ and $\beta$ are variables of the same type (e.g. first-order or second-order), 'Abs' is a term-forming operator that denotes a function from entities of the type of $\alpha$ and $\beta$ to objects, and $\sim$ is an equivalence ${ }^{1}$ relation over entities of that type. Informally, an abstraction principle states that $\alpha$ and $\beta$ have the same abstract just in case they belong to the same equivalence class.

To give an example, [Fre50, 74] formulates an abstraction principle for directions which asserts that two lines $a$ and $b$ have the same direction if and only if they are parallel:

$$
\forall a \forall b(\operatorname{Dir}(a)=\operatorname{Dir}(b) \leftrightarrow a / / b)
$$

Abstraction principles are familiar from neo-Fregeanism in the philosophy of mathematics ([Wri83], [HW01]). Neo-Fregeans attempt to provide a foundation for arithmetic on the basis of Hume's Principle (HP), a (second-level ${ }^{2}$ ) abstraction principle stating that for any two concepts $F$ and $G$, the cardinal number of $F$ is identical with the cardinal number of $G$ just in case $F$ and $G$ are equinumerous, i.e. if and only the $F$ 's and the $G$ 's can be put into one-to-one correspondence:

$$
\forall F \forall G(\#(F)=\#(G) \leftrightarrow F \approx G)
$$

HP is sufficient to derive all the standard axioms of second-order DedekindPeano Arithmetic in fully impredicative ${ }^{3}$ second-order logic with identity and via natural definitions of the arithmetical primitives. This result is now known as Frege's Theorem.

The notion of grounding is similarly familiar from the recent literature in metaphysics (for introduction, see [CS12], [CL12]). Intuitively, not everything that exists is metaphysically on a par; by contrast, the world appears to have some of the features that it has in virtue of it having some others. For example, biological facts arguably obtain in virtue of biochemical facts, and biochemical facts arguably obtain in virtue of micro-physical facts. Many have recently argued that non-causal explanations of this sort should be analysed in terms of a (primitive) notion of metaphysical dependence or grounding, rather than in other (e.g. modal) terms (cf. [Sch09], [Ros10], [Aud12], [Fin12]).

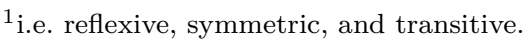

${ }^{2}$ An abstraction principle is first-level if the abstraction operator applies to first-order variables, e.g. (Dir), and second level if the operator applies to second-order variables, e.g HP.

${ }^{3}$ The second-order comprehension axiom is impredicative if the comprehension formula $\phi(x)$ can contain second-order variables, and predicative otherwise.
} 
Abstraction principles and grounding can be combined in a natural way ([Ros10, 177]; [Sch11, 362]). Recall that an abstraction principle states that two items (e.g. lines $a$ and $b$ ) have the same abstract (e.g. the same direction) just in case those items stand in a given equivalence relation (e.g. if and only if they are parallel). It is natural to think that two items have the same abstract in virtue of the fact that those items stand in the relevant relation (e.g. $a$ and $b$ have the same direction because they are parallel). Suppose that at least some of those items are concrete entities, and that their abstracts are, by contrast, mathematical objects, for example numbers. The notion of grounding would then place mathematical entities higher than non-mathematical ones in the metaphysical structure. This might suggest a form of aristotelianism in the philosophy of mathematics, according to which numbers exist and have their properties exclusively in virtue of some non-mathematical facts ${ }^{4}$ ([Ros11]; [Ros16]; [Sch11, 354]; [Don17, 775-6]).

\section{Auto-abstraction}

However, some abstraction principles might be problematic from the point of view of the theory of grounding.

The most straightforward way of formulating a ground-theoretic abstraction principle is by means of a conditional with the following form:

$$
\forall \alpha \forall \beta(\operatorname{Abs}(\alpha)=\operatorname{Abs}(\alpha) \rightarrow([\alpha \sim \beta]<[\operatorname{Abs}(\alpha)=\operatorname{Abs}(\beta)]))
$$

(read: if the abstract of $\alpha$ is identical with the abstract of $\beta$, then the fact that $\alpha \sim \beta$ grounds the fact that the abstract of $\alpha=$ the abstract of $\beta$ ), where the symbol ' $<$ ' stands for full ${ }^{5}$, immediate ${ }^{6}$, and strict ${ }^{7}$ grounding between facts ${ }^{8}$. For example, the ground-theoretic version of HP would be as follows:

$$
\forall F \forall G(\#(F)=\#(G) \rightarrow([F \approx G]<[\#(F)=\#(G)])),
$$

(read: if the number of $F$ is identical with the number of $G$, then the fact that $F \approx G$ grounds the fact that the number of $F=$ the number of $G$ ).

Ground-theoretic abstraction principles must be stated as conditionals because grounding is a factive notion, i.e. $[A]<[B]$ entails both that it is the

\footnotetext{
${ }^{4}$ Aristotelianism bears great significance for the discussion whether a form of physicalism, understood as the claim that everything is either physical or grounded in the physical, is true; I am grateful to an anonymous reviewer at Synthese for drawning my attention to this.

${ }^{5}$ A set of facts $\Gamma$ fully grounds a fact $[A]$ if the former provide a completely satisfactory metaphysical explanation of the latter, while $\Gamma$ partially grounds $[A]$ if the former helps grounding the latter.

${ }^{6} \mathrm{~A}$ set of facts $\Gamma$ immediately grounds $[A]$ iff there is no fact "in between" the ground and the grounded; $\Gamma$ mediately grounds $[A]$ otherwise.

${ }^{7}$ A set of facts $\Gamma$ strictly grounds $[A]$ if for any set of facts $\Delta$ that contains $[A]$, there is no fact $[B] \in \Gamma$ that is mediately grounded by $\Delta$.

${ }^{8} \mathrm{We}$ adopt the convention of enclosing full sentences in squared brackets as a device to indicate facts.
} 
case that $A$ and that it is the case that $B$; without the appropriate conditional form, the ground-theoretic version of HP would have the absurd consequence that each concept is equinumerous with any other ([Sch11, p. 362, fn. 18]).

Moreover, GHP does not account for numerical inequalities, i.e. each case in which the numbers of two concepts are not the same. However, HP states that the equinumerosity of two concepts is sufficient and necessary for the identity of the cardinal numbers of those concepts; therefore, it is natural to extend SRP in in this way:

$$
\begin{gathered}
\forall F \forall G(\#(F)=\#(G) \rightarrow([F \approx G]<[\#(F)=\#(G)]) \wedge \\
\#(F) \neq \#(G) \rightarrow([F \not G]<[\#(F) \neq \#(G)]))
\end{gathered}
$$

Finally, GHP has different consequences depending on how finely the numerical identities on the left of HP are individuated. [Don17, 784-5] distinguishes between two views:

(i) $[\#(F)=\#(G)]$ has two concepts, i.e. $F$ and $G$, the cardinality operator, and the identity relation as constituents;

(ii) $[\#(F)=\#(G)]$ have an object, i.e. the cardinal number of $F$ and $G$, and the identity relation as constituents.

Donaldson labels (i) the "fine view" and (ii) the "coarse view"9. If the fine view is adopted, GHP delivers an account of what grounds numerical identities of the form $[\#(F)=\#(G)]$, but fails to explain what grounds numerical identities of the form $[x=y]$. By contrast, if the coarse view is adopted, GHP fails to provide an account of what grounds contingent numerical identities (e.g. the number of Jupiter's moons = the number of the horses pulling the cart; cp. $[$ Fre50, §57]), since those identities would be the same as necessary identities such as $4=4$. Donaldson suggests that the aristotelian should adopt the fine view and that GHP should be modified as follows:

For all $x, y, F, G$, if $x=\#(F)$ and $y=\#(G)$ and $x=y$, then each one of the facts $[\#(F)=\#(G)],[x=\#(F)],[y=\#(G)]$ and $[x=y]$ is fully grounded by $[F \approx G]$;

For all $x, y, F, G$, if $x=\#(F)$ and $y=\#(G)$ and $x \neq y$, then each one of the facts $[\#(F) \neq \#(G)],[x \neq \#(G)],[y \neq \#(F)]$ and $[x \neq y]$ is fully grounded by $[F \not \approx G] .^{10}$

For example, suppose that there are as many species of Flamingo as there are cities in Wales (this exotic example is due to [Don17, 792]). GHP entails that the fact that the number of Flamingo species is the same as the number of Welsh cities is grounded by the fact that those species and those cities can be paired one-to-one. Assume, moreover, that there are exactly six species of

\footnotetext{
${ }^{9}$ An anonymous reviewer at Synthese pointed out to me that since the notion of grounding is notoriously fine-grained, it is natural for the proponent of GHP to adopt a correspondingly fine-grained conception of facts.

${ }^{10}[$ Don 17,785$]$.
} 
Flamingo and exactly six cities in Wales. It would follow that the fact that $6=6$ is grounded by the fact that there are as many species of Flamingo as cities in Wales.

Consider however the following instance of HP, which states that the number of the concept identical with $1,\ulcorner x=1\urcorner$, is identical with itself if and only if the things that are identical with 1 can be put into one-to-one correspondence with themselves:

$$
\#(\ulcorner x=1\urcorner)=\#(\ulcorner x=1\urcorner) \leftrightarrow\ulcorner x=1\urcorner \approx\ulcorner x=1\urcorner
$$

Note two things. First, it is natural to assume that a first-order universal quantification is partially grounded by each one of its instances; we can therefore adopt the following rule (cf. [Fin10, 100]; [Don17, 779]):

UNIVERSAL GROUNDING: If $\forall x \phi(x)$, infer: $\forall x \phi(x)]$ is fully grounded by the set of facts of the form $[\phi(x)]$.

Second, each instance of HP involves first-order universal quantification on its right. Indeed ' $F \approx G$ ' abbreviates the (purely second-order logical) statement that there is a relation $\mathrm{R}$ such that, for each $F$, there is exactly one $G$ to which that $F$ bears R, and, for each $G$, there is exactly one $F$ bearing $\mathrm{R}$ to that $G$ - formally,

$\exists R(\forall x(F(x) \rightarrow \exists ! y(G(y) \wedge R(x, y))) \wedge \forall x(G(x) \rightarrow \exists ! y(F(y) \wedge R(y, x))))^{11}$

Putting these two together, each instance of the right-hand side of HP will therefore be partially grounded by facts concerning the objects that lie within the range of its (first-order) quantifiers.

Here is why (1) is problematic. Following GHP, the left-hand side of (1) is fully grounded by its right-hand side:

$$
[\ulcorner x=1\urcorner \approx\ulcorner x=1\urcorner]<[\#(\ulcorner x=1\urcorner)=\#(\ulcorner x=1\urcorner)]
$$

As seen, the right-hand side of (1) is logical equivalent to a complex fact, namely:

$[\exists R(\forall x(x=1 \rightarrow \exists ! y(y=1 \wedge R(x, y))) \wedge \forall x(x=1 \rightarrow \exists ! y(y=1 \wedge R(y, x))))]$

The fact that there is a relation of one-to-one correspondence between two concept $F$ and $G$ is plausibly grounded, for each such relation R, by the fact that $\mathrm{R}(F, G)$. Let's pick identity as a paradigmatic case of one-to-one correspondence between two concepts; the right-hand side of (1) is fully grounded by:

$$
[\forall x(x=1 \rightarrow \exists ! y(y=1 \wedge x=y)) \wedge \forall x(x=1 \rightarrow \exists ! y(y=1 \wedge y=x))]
$$

Let's consider the first conjunct first (the same considerations apply to the second conjunct as well). Arguably, a conjunction $A \wedge B$ is partially grounded

${ }^{11} ‘ \exists x ! \phi(x)$ ' is defined as: $\exists x(\phi(x) \wedge \forall y(\phi(y) \rightarrow x=y))$. 
by both $A$ and $B$. Following UNIVERSAL GROUNDING, the first conjunct is partially grounded by:

$$
[a=1 \rightarrow \exists ! y(y=1 \wedge a=y)]
$$

for each object $a$ in the range of the first-order quantifiers featuring on the right of (1). It is reasonable to assume that that a (material) conditional $A \rightarrow B$ is fully grounded either by $\neg A$ or by $B ;[a=1 \rightarrow \exists ! y(y=1 \wedge a=y)]$ is therefore grounded by $[\exists ! y(y=1 \wedge a=y)]$. Since the number 1 itself lies within the range of the first-order quantifiers on the right of $(1)$, then $[\exists ! y(y=1 \wedge a=y)]$ is partially grounded by:

$$
[\exists ! y(y=1 \wedge y=1)]
$$

We can also assume, in analogy with UNIVERSAL GROUNDING, that a first-order existential quantification is fully grounded by each one of its instances; $[\exists ! y(y=1 \wedge y=1)]$ is therefore fully grounded by $[1=1 \wedge 1=1]$, and so by $[1=1]$ alone.

Let's introduce the symbol ' $\prec$ ' for partial grounding. As seen, the righthand side of (1) turns out to be partially grounded by [1 $=1]$ :

$$
[1=1] \prec[\ulcorner x=1\urcorner \approx\ulcorner x=1\urcorner]
$$

At the same time, GHP entails that $[\ulcorner x=1\urcorner \approx\ulcorner x=1\urcorner]$ fully grounds the left-hand side of $(1)$, i.e. $[1=1]$ (since the number of the things identical with 1 is 1 itself). Full grounding is usually taken to entail partial grounding ([?, cf.]51]Fine2012); therefore we have that

$$
[\ulcorner x=1\urcorner \approx\ulcorner x=1\urcorner] \prec[1=1]
$$

By transitivity of partial grounding, $[1=1]$ turns out to be partially grounded by itself, contrary to the grounding orthodoxy, according to which partial grounding is acyclic ([Don17, 793]). I shall call this problem auto-abstraction ${ }^{12}$.

I will proceed as follows. In section 3 I start comparing different solutions to the problem. In section $4 \mathrm{I}$ contend that the thesis that the right-hand side of an abstraction principle is (metaphysically) prior to its left-hand side, motivates an independence constraint, and that this constraint leads to predicative restrictions on the acceptable instances of ground-theoretic abstraction principles. In section $5 \mathrm{I}$ argue that auto-abstraction is acceptable unless the left-hand side is essentially grounded by the right-hand side. In section 6 I highlight several parallelisms between auto-abstraction and the puzzles of ground. I finally compare my solution with the strategies listed in section 3.

${ }^{12}$ I owe the label 'auto-abstraction' to Øyestein Linnebo. 


\section{Priority}

On the face of it, there seem to be three ways one might go in order to solve the problem:

(a) restricting on which instances of HP can correspond to legitimate claims of grounding;

(b) giving up on grounding irreflexivity;

(c) weakening UNIVERSAL GROUNDING.

In section 4 I argue that (a) leads directly to (c), so for now I will focus on (a) and (b).

[Don17, 793-4] argues that the proponent of GHP should follow (b), hence giving up on the acyclicity of partial grounding. Cases of symmetric metaphysical dependence plausibly support this solution; compare the following example given by Elizabeth Barnes:

The event WWII contains many smaller events - some insignificant (such as a particular lighting of a cigar by Winston Churchill) some much more significant (such as the evacuation of Dunkirk). And while WWII might have been the same event without that particular lighting of Churchill's cigar, it's plausible that WWII just wouldn't have been the same event without the evacuation at Dunkirk. Without the evacuation at Dunkirk, it literally would have been a different war - - the evacuation is an essential part of the war. But, similarly, we might think that being a part of WWII is essential to the evacuation of Dunkirk. Sure, you could have a duplicate of that event that doesn't take place in the wider context of WWII. But that duplicate isn't the evacuation at Dunkirk - part of what it is to be the evacuation at Dunkirk is to be a part of $\mathrm{WWII}^{13}$.

Donalson's proposal is therefore that each instance of HP corresponds to a legitimate claim of grounding, regardless of whether that instance entails that there are circles of partial grounding ${ }^{14}$.

(a) requires by contrast that we distinguish between legitimate instances of HP, which correspond to claims of grounding, and illegitimate ones, which do not correspond to such claims. There are indeed many cases in which GHP seems both plausible and unproblematic; it is natural to think, for example, that the number of Flamingo species is the same as the number of Welsh cities in virtue of the fact that those species and those cities can be paired one-to-one. One might therefore argue that a a given instance of HP might correspond to a statement of grounding, but only provided that some extra

\footnotetext{
13 [Bar18, 60].

${ }^{14}$ In fact, Donaldson's suggestion on the behalf of the proponent of GHP is that she should both restrict UNIVERSAL GROUNDING and give up on the acyclicity of grounding. Donaldson's proposal is therefore a combination of (b) and (c); however, (c) alone does not prevent the insurgence of auto-abstraction (see below).
} 
conditions are met (where, crucially, those conditions might result in a ban of auto-abstraction $)^{15}$.

Let's briefly consider the pros and cons of these two strategies. An advantage of the first strategy is that the proponent of GHP need not distinguish between instances of HP that correspond to claims of grounding and instances that don't. The cost of adopting (a) is however that this strategy is burdened by a commitment to cycles of partial ground. By contrast, an advantage of the second strategy is that it can be made consistent with the acyclicity of partial grounding; the proponents of (b) might indeed argue that the instances of HP that would give rise to cases of auto-abstraction don't correspond to genuine claims of grounding. At the same time, (b) comes with a substantial cost, namely that of specifying under which conditions an instance of HP corresponds to a claim of grounding.

Which solution should be preferred? I will now argue that this issue can be settled by considerations of metaphysical priority.

The aristotelian plausibly argues that at least in ordinary cases, relations of equinumerosity between concepts are more fundamental than identities between cardinal numbers, and, in particular, that the right-hand side of an instance of HP, which states that the F's and the $G$ 's can be paired one-toone, is metaphysically prior to its left-hand side, which states that that the cardinal numbers of those $F$ 's and those $G$ 's are identical. I will refer to this as the priority thesis:

PRIORITY: The right-hand side of an instance of an abstraction principle is metaphysically prior to its left-hand side.

For example, according to the aristotelian the fact that the species of Flamingo can be paired one-to-one with the cites in Wales might be metaphysically prior to the fact that the number of those species is identical with the number of those cities.

Moreover, the aristotelian is likely to take GHP as a way of specifying PRIORITY. Priority and grounding are indeed two related notions. More precisely, grounding relations are supposed to be anti-reflexive, anti-symmetric, and transitive. Therefore, those relations determine a strict partial order over facts. Assuming that the right-hand side of an instance of HP grounds the lefthand side, the left-hand side is not grounded, in its turn, by the right-hand side.

Let's go back to auto-abstraction. As seen, GHP implies that the left-hand side of (1) is fully grounded by its left-hand side, and, at the same time, that the right-hand side is partially grounded by the left-hand side. Therefore, anyone who accepts that the right-to-left direction of (1) is a legitimate claim of grounding must give up on the anti-symmetry of partial grounding (and, if transitivity holds, on irreflexivity as well). At the same time, anyone who gives up on anti-symmetry plausibly gives up on the connection between grounding and priority; in the words of Barnes,

\footnotetext{
${ }^{15}$ For a similar approach cf. [Lin18, 43, fn. 41], who claims that the right-to-left direction of abstraction principles correspond to "grounding potentials".
} 
relations of priority ... are, insofar as I have any grip on them, plausibly asymmetric. And that is because they need to be asymmetric in order to do the work we want them to do. These are relations that are introduced in an attempt to take us from the derivative (the constructed, the grounded, the non-fundamental) down toward the bedrock (the ultimate grounds, the fundamental, the basic). ... Their asymmetry is built into the work we want them to do - it's part of what they are for $^{16}$.

Let's finally go back to the choice between (a) and (b). A further cost of (b) is that it seemingly forces the proponent of GHP to give up on PRIORITY. (a), by contrast, does not require one to give up on the anti-symmetry of grounding; therefore, PRIORITY seemingly tells in favour of strategy (a) ${ }^{17}$.

\section{Independence}

Under which conditions does an instance of an abstraction principle count as a legitimate claim of grounding?

By assumption, the left-hand side of an AP is fully grounded, if any ${ }^{18}$, by its left-hand side. Auto-abstraction phenomena show, however, then in some cases the right-hand side is partially grounded by the left-hand side, and so that the left-hand side is mediately grounded by itself, in contradiction with PRIORITY. A natural constraint to be imposed is therefore as follows:

INDEPENDENCE: The right-hand side of an instance of an AP must be independent of its left-hand side, i.e. the right-hand side must not be partially grounded by the left-hand side.

Our discussion will proceed in terms of this notion of independence; so, instead of asking:

(Q) Which instances of an AP are legitimate claims of grounding?

we will ask:

$\left(Q^{\prime}\right)$ Which instances of an AP are such that their right-hand side is independent of their left-hand side?

It is natural to formulate possible responses to $\left(Q^{\prime}\right)$ as restrictions on the righthand side of HP. We will consider three increasingly strong implementations of INDEPENDENCE:

\footnotetext{
16 [Bar18, 54].

${ }^{17}$ Alternatively, the aristotelian might argue that it is not the theoretical role of the notion of grounding to lead one from the fundamental to the derivative; cf. e.g. [Bar18, 67-8]. More in general, the aristotelian could consider the notion of fundamentality as a primitive; cf. [Wil14, §IV.i, VI.ii].

${ }^{18} \mathrm{An}$ anonymous reviewer at Synthese pointed out to me that some might take identity facts to be zero-grounded instead, i.e. grounded by an empty set of facts; cf. e.g. [Fin16]. I will not consider this option here.
} 
(A) The right-hand side of an $\mathrm{AP}$ must not make reference to either $\operatorname{Abs}(\alpha)$ or $\operatorname{Abs}(\beta)$;

(B) The right-hand side of an AP must not make reference to abstract objects of the same kind as $\operatorname{Abs}(\alpha)$ and $\operatorname{Abs}(\beta)$;

(C) The right-hand side of an AP must neither refer to nor quantify over abstract objects of the same kind as $\operatorname{Abs}(\alpha)$ and $\operatorname{Abs}(\beta)$.

We will now consider these three implementations in their turn.

(A) requires that the right of an $\mathrm{AP}$ does not refer to the particular abstract objects that are introduced on its left. This requirement can be understood in two ways:

(a) the singular terms ' $\operatorname{Abs}(\alpha)^{\prime}$ and ' $\operatorname{Abs}(\beta)^{\prime}$ ' must not feature on the righthand side;

(b) the right-hand side must not contain any singular term which refers to either $\operatorname{Abs}(\alpha)$ or $\operatorname{Abs}(\beta)$.

As regards (a), consider the following instance of HP:

$$
\#(H)=\#(\ulcorner x=\#(H)\urcorner) \leftrightarrow H \approx\ulcorner x=\#(H)\urcorner
$$

$(\mathrm{H})$ states that the number of the concept $H$ is identical with the number of the things identical with the number of $H$ just in case $H$ and the concept identical with the number of $H$ are equinumerous. $(\mathrm{H})$ is circular because the term '\#(H)' appears on both sides of the biconditional. By contrast, the case of auto-abstraction mentioned above, namely

$$
\#(\ulcorner x=1\urcorner)=\#(\ulcorner x=1\urcorner) \leftrightarrow\ulcorner x=1\urcorner \approx\ulcorner x=1\urcorner,
$$

violates $(b)$, since ' 1 ' and ' $\#(\ulcorner x=1\urcorner)$ ' both refer to the same cardinal number, namely one.

$(B)$ requires in addition that the right-hand side does not refer to abstract objects at all. Consider the following instance of HP:

$\#(\ulcorner x=1 \vee x=82\urcorner)=\#(\ulcorner x=1 \vee x=82\urcorner) \leftrightarrow\ulcorner x=1 \vee x=82\urcorner \approx\ulcorner x=1 \vee x=82\urcorner$

(2) states that the fact that the number of the things identical with either 1 or 82 is identical with itself is grounded by the fact that those things can be paired one-to-one with themselves. Note that both (a) and (b) above are satisfied; however, (2) gives rise to a case of auto-abstraction. There are indeed exactly two things that are identical with either 1 or 82 ; therefore, the lefthand side of $(2)$ is just $[2=2]$. At the same time, the right-hand side of (2) is partially grounded by the identity of each thing that is identical with either 1 or 82 , and so by both $[1=1]$ and $[82=82]$. However, 82 can be defined as the cardinal number of all its predecessors, i.e. as the cardinal number of $\ulcorner x=0 \vee x=1 \vee \ldots x=82\urcorner$. GHP then entails that $[82=82]$ is partially grounded by $[2=2]$, and so that $[2=2]$ is partially grounded by itself.

(C) finally requires that that the right-hand side must not quantify over abstract objects. Recall that a first-order universal quantification is partially 
grounded by each one of their instances. Now consider $[0=0]$. This identity is plausibly grounded by the fact that any concept with no instances is equinumerous with itself. Let's consider the concept non-self-identical, whose extension is (necessarily) empty. The corresponding instance of HP is:

$$
\#(\ulcorner x \neq x\urcorner)=\#(\ulcorner x \neq x\urcorner) \leftrightarrow\ulcorner x \neq x\urcorner \approx\ulcorner x \neq x\urcorner
$$

The fact expressed on the right-hand side of (0), namely

$[\exists R(\forall x(x \neq x \rightarrow \exists ! y(y \neq y \wedge R(x, y))) \wedge \forall x(x \neq x \rightarrow \exists ! y(y \neq y \wedge R(y, x))))]$

is partially grounded by

$$
[\forall x(x \neq x \rightarrow \exists ! y(y \neq y \wedge S(x, y))) \wedge \forall x(x \neq x \rightarrow \exists ! y(y \neq y \wedge S(y, x)))]
$$

for each one-to-one correspondence relation $S$ between non-self-identical and itself. Since this latter fact involves a first-order universal quantification, it is partially grounded by

$$
[1 \neq 1 \rightarrow \exists ! y(y \neq 1 \wedge S(x, y))) \wedge 1 \neq 1 \rightarrow \exists ! y(y \neq y \wedge S(y, 1))]]
$$

Both the conjuncts of this fact are material conditionals with false antecedents. Therefore, the right-hand side of $(0)$ is partially grounded by $[1 \neq 1]$, which is plausibly grounded in its turn by $[1=1]$. Therefore, GHP entails that $[0=0]$ (or, for that matter, any other numerical identity) is partially grounded by [1 $=1$ ] (and by any other numerical identity whatsoever).

In order to avoid this regress, Donaldson relies on a form of restricted quantification. He writes

$$
(\forall x: F(x))(G(x)) \quad(\exists x: F(x))(G(x))
$$

for 'every $x$ which is an $F$ is a $G$ ' and 'some $x$ which is an $F$ is a $G$ ' respectively ${ }^{19}$. He then introduce the following rule for restricted first-order universal quantification:

UNIVERSAL GROUNDING*: If $(\forall x: F(x))(G(x))$, infer: $[(\forall x$ : $F(x))(G(x))]$ is fully grounded by the set of facts of the form $[(G(x))]$, where $x$ is an $F$.

Finally, Donaldson defines 'Simple $F, G$ ' a relation $\mathrm{R}(x, y)$ such that the first relatum is an $F$ and the second relatum is a $G$; he then restates GHP as follows:

For all $x, y, F, G$, if $x=\#(F)$ and $y=\#(G)$ and $x=y$, then each one of the facts $[\#(F)=\#(G)],[x=\#(F)],[y=\#(G)]$ and $[x=y]$ is fully grounded by $\left[\left(\exists R: \operatorname{Simple}_{F, G}(R)(F \approx G)\right]\right.$

\footnotetext{
${ }^{19}$ As Donaldson points out, "when you utter ' $\forall x$ : Owl $\left.(x)\right)$ (Wise $(x)$ )' you say of the owls that they are wise. When you utter ' $\forall x(\mathrm{Owl}(x) \rightarrow$ Wise $(x))$ ', you say, of each thing that exists, that if it is an owl then it is wise" ([Don17, 788-9; modified for exposition purposes]).
} 
Following UNIVERSAL GROUNDING* and Donaldson's restatement of GHP, $[0=0]$ is grounded by

$$
[\exists R((\forall x x \neq x)(\exists ! y \neq y)(R(x, y)) \wedge(\forall x x \neq x)(\exists ! y: y \neq y)(R(y, x))))]
$$

Since nothing is non-self-identical, Donaldson contends that $[0=0]$ is zerogrounded, i.e. grounded in an empty set of facts ([Don17, 789-90]).

However, UNIVERSAL GROUNDING* does not avoid auto-abstraction completely. Consider for example the following cases:

Consider the [concept] of being one of Celia's favourite things. There are five things which have this property, one of which is the number five. Now consider the [concept] of being a prime number less than six. There are three things which have this property, one of which is the number three ${ }^{20}$.

Even once UNIVERSAL GROUNDING* is taken on board, Donaldson's modification of GHP would imply in this cases that $[5=5]$ and $[3=3]$ are partially grounded by themselves.

Donaldson notes that auto-abstraction emerges when the number of a concept $F$ is itself an $F$. He calls 'autoarithmetic' the concepts with this property. So why not restrict GHP to the concepts that are not autoarithmetic in Donaldson's sense? Note however that the concept identical with $1 \vee$ identical with 82 is not autoarithmetic, but, as seen above, it still gives rise to a case of auto-abstraction ${ }^{21}$.

Generalized auto-abstraction is in fact due to the impredicativity exhibited by abstraction principles like HP. An abstraction principle is impredicative if the objects that are purportedly denoted by the terms that feature on its left-hand side are included in the range of some quantifier that occurs on its right-hand side, and predicative otherwise. As long as impredicativity is allowed, it will always be an open possibility that some identities between abstract objects are at least partially grounded by themselves. Therefore, a way of avoiding auto-abstraction (and circularity in general) would be to restrict GHP to the predicative instances of HP, hence adopting the strongest one of the three implementations that we examined in this section, namely

PREDICATIVITY: The right-hand side of a legitimate instance of HP must not quantify over cardinal numbers.

Compliance with PREDICATIVITY ensures that GHP complies with INDEPENDENCE, and so with PRIORITY as well.

Note that PREDICATIVITY amounts to a qualified variant of strategy (c), namely:

(c) weakening UNIVERSAL GROUNDING.

\footnotetext{
$20[$ Don17, 786].

${ }^{21}$ An anonymous reviewer at Synthese noted that Donaldson's 'Celia' case really shows that it is a contingent matter which instances of the right-hand side of HP are independent, in the relevant sense, of their left-hand side; I think that this remark further supports the solution to the problem of auto-abstraction that I suggest in the next section.
} 
In particular, the proponent of GHP might retain UNIVERSAL GROUNDING as it is, while she adopts PREDICATIVITY and the other restrictions listed by Donaldson to the effect that the universal quantifiers on the right of HP are grounded only in their predicative instances. Doing so would allow one to implement strategy (a), thus giving up on the claim that each instance of HP corresponds to a genuine claim of grounding, without having to embark on strategy (b), thus preserving the irreflexivity of grounding.

As seen, the aristotelian is plausibly committed to PRIORITY. However, there is a sense in which PREDICATIVITY would pull the rug under the aristotelian's feet. In light of Frege's Theorem, according to which all the standard axioms of Peano Arithmetic can be derived from HP alone, GHP promises "to yield an account of what grounds a very wide variety of arithmetical facts" ([Don17, 784]). However, predicative Frege Arithmetic, i.e. the second-order logical theory with predicative Hume's Principle as its sole non-logical axiom, falls short of proving full Peano Arithmetic, but proves only Robinson Arithmetic Q instead. Therefore, if the aristotelian is willing to preserve PRIORITY, and so the philosophical significance of GHP, then she must give up on her convincement that GHP can provide a metaphysical foundation for arithmetical truths; vice versa, if GHP must be apt to provide such foundation, then the aristotelian is apparently forced to at least partly drop INDEPENDENCE by giving up on PREDICATIVITY ${ }^{22}$.

\section{Predicativity}

Let's consider (1) again. GHP entails that the left-hand side of (1), namely $1=1$, is partially grounded by itself. However, GHP also implies that $[1=$ $1]$ is grounded in other and independent ways. For example, 1 is the cardinal number of the concept identical with 0 . The corresponding instance of HP is as follows:

$$
\#(\ulcorner x=0\urcorner)=\#(\ulcorner x=0\urcorner) \leftrightarrow\ulcorner x=0\urcorner \approx\ulcorner x=0\urcorner
$$

Following Donaldson's restatement, the right-hand side of $\left(1^{*}\right)$ is grounded by $[0=0]$ alone, which is zero-grounded in its turn. Therefore, unlike $(1),\left(1^{*}\right)$ does not entail that $[1=1]$ is partially grounded by itself.

Note that the equinumerosity of two concepts is a matter of those concepts' being isomorphic. By contrast, the identity of the specific objects falling under those concepts is in a sense irrelevant to the fact that those concepts are equinumeorus. Suppose for example that $F$ is autoarithmetic in Donaldson's sense, and let $n$ be its number. GHP then entails that $[n=n]$ is partially grounded by itself. However, it would always be possible to find a different concept $G$ such that $F \approx G$, and so $n=\#(G)$, but $n$ is not itself a $G$. GHP

\footnotetext{
${ }^{22}$ An option that we do not consider here consists in departing from abstraction principles as conceived of by neo-Fregeans and by Donaldson alike by taking abstraction to expand the domain into a larger one; cf. [Lin18].
} 
would then entail that $[n=n]$ is partially grounded by $[a=a]$ for each $G(a)$, where, crucially, $a \neq n$.

We can therefore distinguish between two cases of auto-abstraction, which I propose to label 'essential' and 'inessential' respectively. Let a fact be essentially grounded in another fact if, provided that there are multiple ways in which the former fact is (fully) grounded, the latter fact invariably helps grounding the former; that is, the grounding fact figures among the grounded fact's grounds for each way in which the grounded fact is (fully) grounded. By contrast, let a fact be inessentially grounded by another fact if even though the latter does ground the former, there are some ways in which the grounded fact is (fully) grounded which does not include that particular grounding fact.

For example, the left-hand side of an instance of an AP is essentially grounded by its right-hand side if the identity $a_{1}=a_{2}$ that features on the left of that instance invariably obtains in virtue of the obtaining of the corresponding equivalence relation $\alpha \sim \beta$ that features on its right. By contrast, the left-hand side is inessentially grounded by the right-hand side if some of the ways in which $a_{1}=a_{2}$ is grounded do not involve $\alpha \sim \beta$. Vice versa, the right-hand side is essentially grounded by the left-hand side if $\alpha \sim \beta$ invariably obtains in virtue of $a_{1}=a_{2}$, and inessentially grounded by the left-hand side otherwise.

Since, by assumption, the right-hand side fully grounds the left-hand side, then if the left-hand side is essentially grounded by the right-hand side, and the right-hand side is essentially grounded by the left-hand side, then the lefthand side is essentially grounded by itself. We will say that the left-hand side is essentially auto-abstracted in this case. By contrast, even if the right-hand side is grounded by the left-hand side, the left-hand side itself is inessentially auto-abstracted if either the right-hand side inessentially grounds the left-hand side, or the left-hand side inessentially grounds the right-hand side.

Given Donaldson's refinements of GHP, moreover, the right-hand side of an instance of HP is grounded by the left-hand side if it is essentially grounded by the left-hand side at all. Suppose indeed that $F \approx G$, and let $n$ be the number of $F$. Then, if $[F \approx G]$ is grounded by $[n=n]$, it will be so because either $F(n)$ or $G(n)$. Since $[F \approx G]$ is fully grounded by the obtaining of some relation of one-to-one correspondence between the $F$ 's and the $G$ 's, any such relation will therefore involve facts about $n$. By contrast the left-hand side of an instance of HP might be inessentially grounded by the right-hand side. For example, if there are exactly six species of Flamingo and six cities in Wales, Donaldson's version of GHP implies that $[6=6]$ is grounded by facts that concern those cities and those species alone. However, other collection of exactly six things, say the collection of six's predecessors including zero, would have done in their place.

I will now argue that this distinction between essential and inessential cases motivates a natural solution to the problem of auto-abstraction.

Suppose that GHP implies the left-hand side of an instance of HP is grounded by itself, but that the relevant numerical identity is not essentially auto-abstracted. In this case, there would be a perfectly good metaphysical ex- 
planation of why the left-hand side holds which does not mention the truth of right-hand side. In this case the relevant identity between cardinal numbers is available independently of the right-hand side. Provided that that same identity is also independently and ultimately grounded by facts that are entirely non-mathematical, the aristotelian can seemingly accept that the left-hand side is grounded by itself without offending against her naturalism, since no matter whether the left-hand side is grounded by itself, it is also grounded in other and independent ways.

Suppose by contrast that the left-hand side is essentially auto-abstracted. Then each way in which the relevant numerical identity is grounded would involve reliance on that identity itself; a fortiori, some facts about numbers would be ultimately grounded in facts involving (the same) numbers, contrary to the aristotelian intuition that mathematical facts are grounded in non-mathematical ones. So while inessential autoabstraction might be tolerated, essential auto-abstraction cannot be accepted without giving up on on aristotelianism altogether.

Recall that if the right-hand side is grounded by the left-hand side, then the right-hand side is essentially grounded by the left-hand side. At the same time, essential auto-abstraction is unacceptable, while inessential auto-abstraction isn't. The proponent of GHP should therefore adopt the following principle:

AUTO-ABSTRACTION PRINCIPLE: Auto-abstraction can be tolerated unless the left-hand side is essentially grounded by the right-hand side.

This principle has consequences for all the cases of auto-abstraction mentioned so far. As seen, $[1=1]$ is ultimately zero-grounded; therefore, (1) turns out to be acceptable. Let's now move to $(2)$. Even if $[2=2]$ is grounded by both $[1=1]$ and $[82=82]$, and this latter is grounded in its turn by [2=2], 2 is the cardinal number of the concept identical with $0 \vee$ identical with 1 , and so GHP entails that $[2=2]$ is grounded by $[1=1]$ and $[0=0]$ (and so by $0=0$ alone). We can therefore deem (2) as acceptable as well. Finally, even if Celia has exactly five favourite things, and the cardinal number five is one of them, $[5=5]$ is also grounded in facts involving for example all the predecessors of five, which do not include five itself; similarly, $[3=3]$ is grounded by $[2=2]$, $[1=1]$, and $[0=0]$, and so by $[0=0]$ alone.

The AUTO-ABSTRACTION PRINCIPLE has also consequences for predicativity in general. As seen, the independence constraint motivates predicative restrictions on the acceptable instances of GHP. Note however that each (finite) cardinal number can be defined as the cardinal number of all its predecessors including zero. As seen, 1 can be defined as the cardinal number of the concept identical with 0 , and 2 can be defined as the number of identical with $0 \vee$ identical with 1; 3 can be defined as the number of identical with $0 \vee$ identical with $1 \vee$ identical with 2, and so on. Given Donaldson's refinements of GHP, each finite cardinal number is grounded by $[0=0]$, and so ultimately zero-grounded. Since quantification on those numbers on the left of HP is sufficient to derive the standard axioms of Dedekind-Peano Arith- 
metic, the AUTO-ABSTRACTION PRINCIPLE restores GHP's promise to deliver an account of the metaphysical foundations of "a very wide variety" of arithmetical facts.

Finally, the AUTO-ABSTRACTION PRINCIPLE has consequences for aristotelianism at large. Recall that the aristotelian is plausibly committed to the thesis that the right-hand side of an instance of HP is metaphysically prior to the left-hand side. I argued above that the AUTO-ABSTRACTION PRINCIPLE entails that quantification over at least finite cardinal numbers should be tolerated, in violation of PREDICATIVITY. Since PREDICATIVITY is violated, the AUTO-ABSTRACTION PRINCIPLE makes room for cases in which the right-hand side is grounded by the left-hand side, in violation of INDEPENDENCE. The violation of INDEPENDENCE leads to the straightforward violation of PRIORITY: provided that the left-hand side is not essentially auto-abstracted, some numerical identities will be grounded by themselves. However, even though in those cases the right-hand is not independent of, and consequently prior to, the left-hand side, there is a different kind of independence that is preserved, that this, the left-hand side would still be available independently of the right-hand side. If the AUTO-ABSTRACTION PRINCIPLE is enforced, however, to allow those apparently problematic instances of HP would not result in more arithmetical identities than the ones that we would have got hadn't we allowed such instances ${ }^{23}$

\section{Auto-abstraction and the Puzzles of Ground}

Recall that one of the three strategies, (c), consists in weakening UNIVERSAL GROUNDING.

UNIVERSAL GROUNDING might indeed appear to some as problematic in itself. For example, let $\mathrm{F}$ be the fact that everything exists. The fact that $\mathrm{F}$ exists is plausibly grounded by everything's existing, since $\mathrm{F}$ is the fact that everything exists. At the same time, UNIVERSAL GROUNDING entails that everything exists partly in virtue of F's existing. Therefore, everything exists partially in virtue of everything's existing, in contradiction with grounding irreflexivity ${ }^{24}$.

Fine contends that puzzles like this highlight "a conflict between a deeply entrenched logical view, on the one side, and extremely plausible metaphysical views, on the other side" ([Fin10, 105]). As regards logic, it is a standard logical theorem that everything exists. As regards metaphysics, Fine mentions two views, namely Complex Ground, i.e. the view that every logically complex truth should have a ground, and Classicality, i.e. the view that those ground,

\footnotetext{
${ }^{23}$ Note the solution to the problem of auto-abstraction that I developed in this section bears close resemblance to Wright's defence of the impredicativity of HP; cf. [Wri97], [Wri98], [Wri99].

${ }^{24}$ Even though Fine's own version of the puzzles of ground rests on a number of assumption, [Kra13] formulates the same puzzle in such a way as a contradiction is derived from irreflexivity and (an higher-order version of) UNIVERSAL GROUNDING alone.
} 
if any, should be in conformity with the classical truth-conditions; together, those two views support UNIVERSAL GROUNDING. Fine then proceeds to examine various ways in which both classical logic and the logic of ground can be weakened in order to solve the puzzles.

Fine briefly considers whether irreflexivity could be dropped instead; however he claims that

there is ... a plausible demand on ground or explanation that we are unable to evade. Given a truth that stands in need of explanation, one naturally supposes that it should have a "completely satisfactory" explanation, one that does not involve cycles and terminates in truths that do not stand in need of explanation ${ }^{25}$.

[Lit15, 449] notes that "on the assumption that every truth has a completely satisfactory explanation, it follows that this notion of ground satisfies Noncircularity". Suppose indeed that there is a putative explanation of $A$ in terms of $A$ itself; arguably, the explanation thereby provided would not be "completely satisfactory", since it contains a cycle involving $A$.

Litland grants that, even if $A$ is partly explained by $A, A$ might admit of an independent explanation - that is, an explanation that does not involve $A$ itself. Inessential auto-abstraction is of course a case like this; for example, even though the right-hand side of (1) grounds its left-hand side, there is also an independent explanation of why $1=1$ holds. Litland argues however that "if one has already given a satisfactory explanation of why $A$ is the case, one does not in any way improve from that explanation by going on from $A$ to explain it again" ([Lit15, 499-500, modified for exposition purposes]). One might argue that once $1=1$ has been given a satisfactory explanation, there is not point in considering (1) as a claim of grounding. At the same time, $1=1$ still explain why $\#(\ulcorner x=1\urcorner)=\#(\ulcorner x=1\urcorner)$. So one might restrict Donaldson's restatement of GHP in such a way that, if $F \approx G$ and $n=\#(F)$, but $n=n$ is already available, then $[F \approx G]$ grounds $[\#(F)=\#(G)]$ but not $[n=n]$.

By contrast, both [Cor13] and [Woo18] give up on irreflexivity. Correia suggests that if some independently plausible views about what grounds what, in conjunction with the equally plausible principle that grounding is transitive, entail that grounding is non-reflexive, then we should consider these cases as counterexamples to grounding irreflexivity ([Cor13, 55]).

Woods claims that "grounding is not irreflexive, but it is substantively irreflexive - it is never the case that something is non-vacuously grounded in itself", where some facts occur vacuously among the grounds of some other fact if "their particular content does no substantive work in grounding the grounded fact" ([Woo18, 2]). For example, the fact that everything exists, F, vacuously grounds everything's existing because any other fact would have done in place of F. Woods argues that while circles of non-vacuous grounding are in principle unacceptable, reflexive vacuous ground can be accepted without offending against the intuition that nothing (non-vacuously) explains itself.

${ }^{25}[$ Fin10, 105]. 
Woods's notion of vacuous grounds bears close resemblance to my notion of inessential auto-abstraction. Note that the RHS does not occur vacuously in the grounds of the left-hand side in Woods sense, since not any fact whatsoever would do in place of the equinumerosity of $F$ and $G$. For that matter, not even any equinumerosity fact whatsoever would do in place of a particular instance of the right-hand side: for example, even if Celia's five favourite things are equinumerous with the predecessors of 6 including zero, this fact does not ground $[6=6]$. However, there is a restricted sense in which the right-hand side occurs vacuously among the grounds of the left-hand side, namely with respect to each concept equinumerous with one of the concepts involved in $[F \approx G]$. For example, $[6=6]$ is grounded by the fact that there are as many species of Flamingo as there are cities in Wales; however, $[6=6]$ is also grounded by the fact that those species are equinumerous with the $G$ 's, for any $G$ with exactly six instances.

Moreover, [Fin10, 104] suggests that

as a general matter $A$ would not be a ground, either immediate or mediate, for $A$ but there might be special cases in which $A$ in one capacity, so to speak, was a ground for $A$ in another capacity.

Woods claims that the notion of vacuous ground helps clarifying how "special cases" of reflexive grounding are possible, since vacuous occurences of a fact in its own grounds are cases in which a fact "in one capacity", and specifically in a guise such that the particular content of that fact is not relevant to the grounded fact, grounds the same fact "in another capacity", and specifically in a guise such that its content does matter. My notion of inessential autoabstraction is similarly close to Fine's dictum. Recall indeed that even if $n=$ $\#(F)$, the identity of every particular $F$-thing is irrelevant to $[n=n]$. The corresponding instance of GHP would just imply that $[n=n]$ is grounded in facts involving collections of exactly $n$ things, no matter whether some of those collections contain the number $n$ itself.

Finally, [Lov19] takes the (reflexive) notion of weak ground as a primitive ${ }^{26}$, and derives from it principles for strict ground (roughly: $A$ strictly grounds $B$ if $A$ weakly grounds $B$ and $B$ not even weakly helps grounding $A$ ). This leads to a neat and unified solution to most of the puzzles of ground explored in the literature.

As Fine notes, the puzzles of ground require that one reaches a "reflective equilibrium" between principles that are individually plausible but which are in conflict with each other ([Fin10, 97]). Equilibrium can be achieved in several different ways. However, I think that there is a principled reason to drop grounding irreflexivity (as I suggest in this paper) rather than UNIVERSAL GROUNDING or even classical logic, that is, that classical logic and also UNIVERSAL GROUNDING enjoy an independent plausibility, while irreflexivity is supported only by paradigmatic cases of grounding. If some of those cases entail that grounding is non-irreflexive, then the most conservative modification of one's metaphysical view would be to give up on irreflexivity.

${ }^{26}$ Similarly to [Fin12]. 
Finally, it worth noting that unlike all the extant approaches to the puzzles of ground, I propose a restriction on the acceptable instances of groundtheoretic abstraction principles. This isn't what people usually do in the debate on the puzzles of ground, since none of those puzzles officially involves abstraction principles. This also means that my solution is less general than at least most of the proposed solutions to the puzzles of ground. However, this paper's aim was not to give a general solution to those puzzles, but only to suggest what the proponents of GHP, and the aristotelian more in general, should think about auto-abstraction ${ }^{27}$.

\section{Conclusions}

Let me end up by comparing my solution with strategies (a) and (b). Under which conditions is auto-abstraction acceptable? Strategy (a) requires that ground-theoretic abstraction principles are restricted in such a way as to avoid any case of auto-abstraction whatsoever. Against (a), I argued that autoabstraction is acceptable unless the left-hand side is essentially grounded by the left-hand side. By contrast, strategy (b) requires to give up on irreflexivity altogether. Against (b), I argue that even once irreflexivity is dropped, cases of essential auto-abstraction remain problematic.

\section{References}

[Aud12] Paul Audi. Grounding: Toward a theory of the in-virtue-of relation. Journal of Philosophy, 109(12):685-711, 2012.

[Bar18] Elizabeth Barnes. Symmetric dependence. In Ricki Leigh Bliss and Graham Priest, editors, Reality and Its Structure, pages 50-69. 2018.

[CL12] Michael J. Clark and David Liggins. Recent work on grounding. Analysis Reviews, 72(4):812-823, 2012

[Cor13] Fabrice Correia. Logical grounds. Review of Symbolic Logic, (1):1-29, 2013.

[CS12] Fabrice Correia and Benjamin Schnieder. Grounding: An opinionated introduction. In Fabrice Correia and Benjamin Schnieder, editors, Metaphysical Grounding: Understanding the Structure of Reality, page 1. Cambridge University Press, 2012.

[Don17] Thomas Donaldson. The (metaphysical) foundations of arithmetic? Nôus, 51(4):775-801, 2017.

[Fin10] Kit Fine. Some puzzles of ground. Notre Dame Journal of Formal Logic, 51(1):97$118,2010$.

[Fin12] Kit Fine. Guide to ground. In Fabrice Correia and Benjamin Schnieder, editors, Metaphysical Grounding, pages 37-80. Cambridge University Press, 2012.

[Fin16] Kit Fine. Identity criteria and ground. Philosophical Studies, 173(1):1-19, 2016.

[Fre50] Gottlob Frege. The Foundations of Arithmetic: A Logico-Mathematical Enquiry Into the Concept of Number. Northwestern University Press, 1950. Transl. by J. Austin.

[HW01] Bob Hale and Crispin Wright. The Reason's Proper Study: Essays Towards a Neo-Fregean Philosophy of Mathematics. Oxford: Clarendon Press, 2001.

[Kra13] Stephan Kramer. A simpler puzzle of ground. 2013.

\footnotetext{
${ }^{27}$ The last two sections are offered as a piece of conceptual engineering: we should conceive of grounding as to make room for cases of reflexive partial ground.
} 
[Lin18] Øystein Linnebo. Thin Objects: An Abstractionist Account. Oxford: Oxford University Press, 2018.

[Lit15] Jon Erling Litland. Grounding, explanation, and the limit of internality. Philosophical Review, 124(4):481-532, 2015.

[Lov19] Adam Lovett. The puzzles of ground. Philosophical Studies, pages 1-24, 2019.

[Ros10] Gideon Rosen. Metaphysical dependence: Grounding and reduction. In Bob Hale and Aviv Hoffmann, editors, Modality: Metaphysics, Logic, and Epistemology, pages 109-36. Oxford University Press, 2010.

[Ros11] Gideon Rosen. The reality of mathematical objects. In John Polkinghorne, editor, Meaning in Mathematics, pages 113-31. Oxford University Press, 2011.

[Ros16] Gideon Rosen. Mathematics and metaphysical naturalism. In Kelly Clark, editor, The Blackwell Companion to Naturalism, pages 277-88. Wiley Blackwell, 2016.

[Sch09] Jonathan Schaffer. On what grounds what. In David Manley, David J. Chalmers, and Ryan Wasserman, editors, Metametaphysics: New Essays on the Foundations of Ontology, pages 347-383. Oxford University Press, 2009.

[Sch11] Robert Schwartzkopff. Numbers as ontologically dependent objects hume's principle revisited. Grazer Philosophische Studien, 82(1):353-373, 2011.

[Wil14] Jessica M. Wilson. No work for a theory of grounding. Inquiry: An Interdisciplinary Journal of Philosophy, 57(5-6):535-579, 2014.

[Woo18] Jack Woods. Emptying a paradox of ground. Journal of Philosophical Logic, 47(4):631-648, 2018

[Wri83] Crispin Wright. Frege's Conception of Numbers as Objects. Aberdeen University Press, 1983.

[Wri97] Crispin Wright. On the philosophical significance of frege's theorem. In Richard G. Heck, editor, Language, Thought, and Logic: Essays in Honour of Michael Dummett, pages 201-44. Oxford University Press, 1997.

[Wri98] Crispin Wright. On the harmless impredicativity of $n=($ 'hume's principle'). In Matthias Schirn, editor, The Philosophy of Mathematics Today, pages 339-68. Clarendon Press, 1998.

[Wri99] Crispin Wright. Is hume's principle analytic? Notre Dame Journal of Formal Logic, 40(1):6-30, 1999. 